Louisiana State University

LSU Digital Commons

$5-10-2013$

\title{
Host star properties and transit exclusion for the HD 38529 planetary system
}

\author{
Gregory W. Henry \\ Tennessee State University \\ Stephen R. Kane \\ National Aeronautics and Space Administration \\ Sharon X. Wang \\ Pennsylvania State University \\ Jason T. Wright \\ Pennsylvania State University \\ Tabetha S. Boyajian \\ Yale University
}

See next page for additional authors

Follow this and additional works at: https://digitalcommons.Isu.edu/physics_astronomy_pubs

\section{Recommended Citation}

Henry, G., Kane, S., Wang, S., Wright, J., Boyajian, T., Von Braun, K., Ciardi, D., Dragomir, D., Farrington, C., Fischer, D., Hinkel, N., Howard, A., Jensen, E., Laughlin, G., Mahadevan, S., \& Pilyavsky, G. (2013). Host star properties and transit exclusion for the HD 38529 planetary system. Astrophysical Journal, 768 (2) https://doi.org/10.1088/0004-637X/768/2/155 


\section{Authors}

Gregory W. Henry, Stephen R. Kane, Sharon X. Wang, Jason T. Wright, Tabetha S. Boyajian, Kaspar Von Braun, David R. Ciardi, Diana Dragomir, Chris Farrington, Debra A. Fischer, Natalie R. Hinkel, Andrew W. Howard, Eric Jensen, Gregory Laughlin, Suvrath Mahadevan, and Genady Pilyavsky 
Swarthmore College

Works

$5-10-2013$

\section{Host Star Properties And Transit Exclusion For The HD 38529 Planetary System}

G. W. Henry

S. R. Kane

S. X. Wang

T. S. Boyajian

K. Von Braun

See next page for additional authors

Follow this and additional works at: https://works.swarthmore.edu/fac-physics

Part of the Astrophysics and Astronomy Commons

Let us know how access to these works benefits you

\section{Recommended Citation}

G. W. Henry, S. R. Kane, S. X. Wang, T. S. Boyajian, K. Von Braun, D. R. Ciardi, D. Dragomir, C. Farrington, D. A. Fischer, N. R. Hinkel, A. W. Howard, Eric L.N. Jensen, G. Laughlin, S. Mahadevan, and G. Pilyavsky. (2013). "Host Star Properties And Transit Exclusion For The HD 38529 Planetary System". Astrophysical Journal. Volume 768, Issue 2. DOI: 10.1088/0004-637X/768/2/155

https://works.swarthmore.edu/fac-physics/45

This work is brought to you for free by Swarthmore College Libraries' Works. It has been accepted for inclusion in Physics \& Astronomy Faculty Works by an authorized administrator of Works. For more information, please contact myworks@swarthmore.edu. 


\section{Authors}

G. W. Henry, S. R. Kane, S. X. Wang, T. S. Boyajian, K. Von Braun, D. R. Ciardi, D. Dragomir, C. Farrington, D. A. Fischer, N. R. Hinkel, A. W. Howard, Eric L.N. Jensen, G. Laughlin, S. Mahadevan, and G. Pilyavsky 


\title{
HOST STAR PROPERTIES AND TRANSIT EXCLUSION FOR THE HD 38529 PLANETARY SYSTEM
}

\author{
Gregory W. Henry ${ }^{1}$, Stephen R. KAnE ${ }^{2}$, Sharon X. WAng ${ }^{3,4}$, Jason T. Wright ${ }^{3,4}$, Tabetha S. Boyajian ${ }^{5}$, \\ Kaspar von Braun ${ }^{2,6}$, David R. Ciardi ${ }^{2}$, Diana Dragomir ${ }^{7}$, Chris Farrington ${ }^{8}$, Debra A. Fischer ${ }^{5}$, Natalie R. Hinkel ${ }^{2}$, \\ Andrew W. Howard ${ }^{9}$, Eric Jensen ${ }^{10}$, Gregory Laughlin ${ }^{11}$, Suvrath Mahadevan ${ }^{3,4}$, and Genady Pilyavsky ${ }^{3}$ \\ ${ }^{1}$ Center of Excellence in Information Systems, Tennessee State University, 3500 John A. Merritt Blvd., Box 9501, \\ Nashville, TN 37209, USA; gregory.w.henry@gmail.com \\ ${ }^{2}$ NASA Exoplanet Science Institute, Caltech, MS 100-22, 770 South Wilson Avenue, Pasadena, CA 91125, USA \\ ${ }^{3}$ Department of Astronomy and Astrophysics, Pennsylvania State University, 525 Davey Laboratory, University Park, PA 16802, USA \\ ${ }^{4}$ Center for Exoplanets \& Habitable Worlds, Pennsylvania State University, 525 Davey Laboratory, University Park, PA 16802, USA \\ ${ }^{5}$ Department of Astronomy, Yale University, New Haven, CT 06511, USA \\ ${ }^{6}$ Max Planck Institut for Astronomy, Königstuhl 17, D-69117, Heidelberg, Germany \\ ${ }^{7}$ Las Cumbres Observatory Global Telescope Network, 6740B Cortona Dr., Suite 102, Goleta, CA 93117, USA \\ ${ }^{8}$ The CHARA Array, Mount Wilson Observatory, Mount Wilson, CA 91023, USA \\ ${ }^{9}$ Institute for Astronomy, University of Hawaii, Honolulu, HI 96822, USA \\ ${ }^{10}$ Department of Physics \& Astronomy, Swarthmore College, Swarthmore, PA 19081, USA \\ ${ }^{11}$ UCO/Lick Observatory, University of California, Santa Cruz, CA 95064, USA \\ Received 2013 February 11; accepted 2013 March 18; published 2013 April 25
}

\begin{abstract}
The transit signature of exoplanets provides an avenue through which characterization of exoplanetary properties may be undertaken, such as studies of mean density, structure, and atmospheric composition. The Transit Ephemeris Refinement and Monitoring Survey is a program to expand the catalog of transiting planets around bright host stars by refining the orbits of known planets discovered with the radial velocity technique. Here we present results for the HD 38529 system. We determine fundamental properties of the host star through direct interferometric measurements of the radius and through spectroscopic analysis. We provide new radial velocity measurements that are used to improve the Keplerian solution for the two known planets, and we find no evidence for a previously postulated third planet. We also present 12 years of precision robotic photometry of HD 38529 that demonstrate the inner planet does not transit and the host star exhibits cyclic variations in seasonal mean brightness with a timescale of approximately six years.
\end{abstract}

Key words: planetary systems - stars: individual (HD 38529) - techniques: photometric - techniques: radial velocities

Online-only material: color figures, machine-readable table

\section{INTRODUCTION}

The study of transiting exoplanets has undergone a remarkable evolution since the first transit detection in HD 209458b (Charbonneau et al. 2000; Henry et al. 2000). Projects such as the Hungarian Automated Telescope Network (Bakos et al. 2004), and SuperWASP (Pollacco et al. 2006) are routinely detecting new transiting planets. Results from the NASA Kepler mission are breaking new ground by discovering planets that are smaller and at longer orbital periods than those accessible from ground-based surveys (Borucki et al. 2011a, 2011b). However, the most important transiting planets for follow-up observations and characterization of atmospheres continue to be those discovered first by the radial velocity (RV) method, because of the bias toward bright host stars. The Transit Ephemeris Refinement and Monitoring Survey (TERMS) seeks to provide more of these opportunities through orbital refinement (Kane et al. 2009), particularly for planets in eccentric orbits with higher transit probabilities (Kane \& von Braun 2008). TERMS also allows the study of long-term variability of the host star such that it can be correlated with the stellar magnetic activity cycle in the context of being a planet-host (Dragomir et al. 2012).

Multi-planet systems present particularly interesting cases since studying the dynamical interaction of the planets is an additional advantage to refining the orbits. One such TERMS target is the planetary system orbiting HD 38529, whose planets have been discovered via precision RV measurements. The inner planet is in a $\sim 14$ day orbit and was discovered by Fischer et al. (2001). The second planet, in a $\sim 6$ year orbit, was discovered by Fischer et al. (2003). The system was further investigated in the context of multi-planet systems by Wittenmyer et al. (2009) and Wright et al. (2009), both of whom provided new RV data and revised orbits for the planets. Benedict et al. (2010) furthered the studies of the system by providing new RV data as well as Hubble Space Telescope astrometry. Their analysis indicated that there may be an additional planet located at an orbital period of $\sim 194$ days, though this was not confirmed by their observations and they encouraged further study to help resolve the issue.

In this paper we present an exhaustive analysis of both the host star and the planets in the HD 38529 system. We performed interferometric observations of the host star using the CHARA Array to determine a direct measurement of the radius, which we compare with that derived from an analysis of high-resolution spectra. We provide new RV data for the system, improving the orbits of the planets and greatly extending the time baseline. Our analysis of the combined data finds no evidence for a third planet located near 194 days. From these new data, we calculate an accurate transit ephemeris and predict the parameters of a potential transit of HD $38529 \mathrm{~b}$. We present 12 years of precision photometry of HD 38529 acquired with an automated photoelectric telescope (APT) at Fairborn Observatory. These observations rule out a transit of the inner planet. Furthermore, we demonstrate that the host star exhibits a cyclic brightness 
Table 1

Stellar Properties of HD 38529

\begin{tabular}{lccl}
\hline \hline Parameter & $\begin{array}{c}\text { Value } \\
\text { Spectroscopic }\end{array}$ & $\begin{array}{c}\text { Value } \\
\text { Interferometric }\end{array}$ & Reference \\
\hline$\theta_{\text {UD }}($ mas $)$ & $\ldots$ & $0.593 \pm 0.016$ & This work (Section 2.1) \\
$\theta_{\text {LD }}($ mas) & $\ldots$ & $0.611 \pm 0.016$ & This work (Section 2.1) \\
Luminosity $\left(L_{\odot}\right)$ & $\ldots$ & $5.777 \pm 0.186$ & This work (Section 2.2) \\
Radius $R_{*}\left(R_{\odot}\right)$ & $2.34 \pm 0.07$ & $2.578 \pm 0.080$ & This work (Sections 3.2 and 2.1) \\
$T_{\text {eff }}(\mathrm{K})$ & $5619 \pm 44$ & $5576 \pm 74$ & This work (Sections 3.2 and 2.2) \\
{$[\mathrm{Fe} / \mathrm{H}]$} & $0.38 \pm 0.03$ & $\ldots$ & This work (Section 3.2) \\
$v \sin i\left(\mathrm{~km} \mathrm{~s}^{-1}\right)$ & $3.20 \pm 0.50$ & $\ldots$ & This work (Section 3.2) \\
$\log g$ & $3.83 \pm 0.06$ & $\ldots$ & This work (Section 3.2) \\
Mass $M_{*}\left(\mathrm{M}_{\odot}\right)$ & $1.36 \pm 0.02$ & $\ldots$ & This work (Section 3.2) \\
Age $(\mathrm{Gyr})$ & $4.45 \pm 0.23$ & $\ldots$ & This work (Section 3.2) \\
\hline
\end{tabular}

Notes. For details, see Sections 2 and 3.2.

variation on a timescale of approximately six years which is correlated with the S-index derived from our Keck I spectra.

\section{FUNDAMENTAL STELLAR PARAMETERS}

\subsection{Stellar Radius}

HD 38529 was observed during three nights in 2012 November using the Georgia State University Center for High Angular Resolution Astronomy (CHARA) interferometric array (ten Brummelaar et al. 2005). Our observational methods and strategy are described in detail in von Braun et al. (2012) and Boyajian et al. (2012b) and references therein. We used two of CHARA's longest baselines (S1E1 and E1W1) to perform our observations in $H$-band with the CHARA Classic beam combiner (Sturmann et al. 2003; ten Brummelaar et al. 2005) in single-baseline mode. We obtained 3, 1, and 2 observations (brackets) during the nights of 2012 November 3, 4, and 12, each of which contains approximately 2.5 minutes of integration and 1.5 minutes of telescope slewing per object (target and calibrator). To remove the influence of atmospheric and instrumental systematics, interferometric observations consist of bracketed sequences of object and calibrator stars, chosen to be near-point-like sources of similar brightness as HD 38529 and located at small angular distances from it. We originally used both HD 37077 and HD 36777 as calibrators but eliminated the latter due to the presence of a second fringe packet in each observation, indicating that HD 36777 may be an unresolved binary.

To protect against unknown systematics in interferometric data, we ordinarily require the use of at least two calibrators, two baselines, and data obtained during at least two nights (Boyajian et al. 2012a, 2012b; von Braun et al. 2011a, 2011b, 2012). Thus, we added 12 archival CHARA $K$-band brackets obtained in 2005 using the S1E1 baseline and HD 43318 as calibrator, published by Baines et al. (2008).

The uniform disk and limb-darkened angular diameters $\left(\theta_{\mathrm{UD}}\right.$ and $\theta_{\mathrm{LD}}$, respectively; see Table 1) are found by fitting the calibrated visibility measurements (Figure 1) to the respective functions for each relation. These functions may be described as $n$ th-order Bessel functions of the angular diameter of the star, the projected distance between the two telescopes, and the wavelength of observation (see Equations (2) and (4) of Hanbury Brown et al. 1974). Visibility is the normalized amplitude of the correlation of the light from two telescopes. It is a unitless number ranging from 0 to 1 , where 0 implies no correlation and 1 implies perfect correlation. An unresolved source would have a perfect correlation of 1.0 independent of the distance between

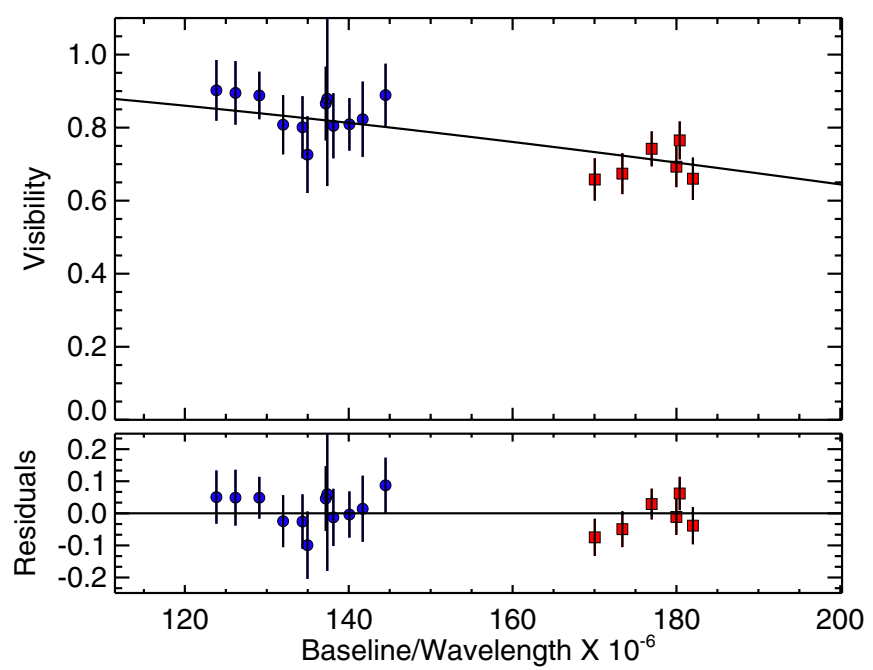

Figure 1. Calibrated visibility observations along with the limb-darkened angular diameter fit for HD 38529 (top panel) along with the fractional residuals around the fit (bottom panel). The blue round points are the $K$-band data, and the red squares are the $H$-band data. The $K$-band data from Baines et al. (2008) were taken with a shorter effective baseline than our new $H$-band data (due to the difference in wavelength). The reduced $\chi^{2}$ of this fit is 0.49 . For more details, see Section 2.1 .

(A color version of this figure is available in the online journal.)

the telescopes (baseline). A resolved object will show a decrease in visibility with increasing baseline length. The shape of the visibility versus baseline is a function of the topology of the observed object (the Fourier Transform of the object's shape). For a uniform disk this function is a Bessel function, and for this paper, we use a simple model for limb-darkening variation of a uniform disk. The visibility of any source is reduced by a nonperfect interferometer, and the point-like calibrators are needed to calibrate out the loss of coherence caused by instrumental effects.

We use the linear limb-darkening coefficient $\mu_{H}=0.362$ from the ATLAS models in Claret (2000) for stellar $T_{\text {eff }}=$ $5500 \mathrm{~K}$ and $\log g=4.0$ to convert from $\theta_{\mathrm{UD}}$ to $\theta_{\mathrm{LD}}$. The uncertainties in the adopted limb darkening coefficient amount to $0.2 \%$ when modifying the adopted gravity by 0.5 dex or the adopted $T_{\text {eff }}$ by $200 \mathrm{~K}$, well within the errors of our diameter estimate.

Our interferometric measurements yield the following values for HD 38529's angular diameters: $\theta_{\mathrm{UD}}=0.593 \pm 0.016$ milliarcseconds (mas) and $\theta_{\mathrm{LD}}=0.611 \pm 0.016$ mas (Table 1 ). Combined with the direct distance measurement from van 
Leeuwen (2007) of $39.277 \pm 0.617 \mathrm{pc}$, we derive a stellar radius for HD 38529 of $2.5780 \pm 0.0795 R_{\odot}$, which is consistent with $2.44 \pm 0.22 R_{\odot}$ calculated by Baines et al. (2008).

\subsection{Stellar Effective Temperature and Luminosity}

To calculate HD 38529's effective temperature and luminosity, we produce a spectral energy distribution (SED) fit based on the spectral templates in Pickles (1998) to photometry from Johnson et al. (1966), Cousins (1962), Argue (1966), Mermilliod (1986), Hauck \& Mermilliod (1998), Olsen (1993), Cutri et al. (2003), McClure \& Forrester (1981), and Golay (1972). We furthermore use the distance calculated in van Leeuwen (2007) and set interstellar reddening to zero.

From the SED fit, we calculate the value of HD38529's stellar bolometric flux to be $F_{\mathrm{BOL}}=(12.02 \pm 0.084) \times$ $10^{-8} \mathrm{erg} \mathrm{cm}^{-2} \mathrm{~s}^{-1}$ and, consequently, its luminosity $L=$ $5.777 \pm 0.186 L_{\odot}$. The calculated effective temperature for HD 38529 is $T_{\text {eff }}=5576 \pm 74 \mathrm{~K}$ (see Table 1$)$.

\section{REFINING THE PLANETARY ORBITS}

Here we present the new RV data and the revised orbital solution for the HD 38529 system. We combine this with the derived host star properties to determine an accurate transit ephemeris for HD 38529 b.

\subsection{Spectra Acquisition}

The RV data for HD 38529 presented here comprise 436 measurements and were acquired from three instruments/telescopes: the High Resolution Spectrograph (Tull 1998) on the Hobby-Eberly Telescope (HET), the Hamilton Echelle Spectrograph (Vogt 1987) on the $3.0 \mathrm{~m}$ Shane Telescope at Lick Observatory, and the HIRES echelle spectrometer (Vogt et al. 1994) on the $10.0 \mathrm{~m}$ Keck I telescope. Shown in Table 2 are a subset of the full data set, available in the electronic version of this paper. The fourth column in Table 2 indicates the six independent data sets that form the combined data, two each from HET, Lick, and Keck. The data sources are as follows: (1) HET data from Benedict et al. (2010); (2) new HET data presented here; (3) Keck data from Wright et al. (2009); (4) Keck data from Wright et al. (2009) before 2009 September 15, new Keck data presented here thereafter; (5) Lick data from Wright et al. (2009); (6) Lick data from Wright et al. (2009) before 2009 September 15, new Lick data presented here thereafter. The division of the Lick data into two separate data sets is necessitated by a change in the dewar resulting in different CCD response characteristics. The offsets between these data sets are accounted for in the Keplerian orbital fitting described below.

\subsection{SME Analysis}

For additional insight into the properties of the host star, we used Spectroscopy Made Easy (SME) (Valenti \& Piskunov 1996) to fit high-resolution Keck spectra of HD 38529. The methodology of this technique, including application of the wavelength intervals and line data, are described in more detail by Valenti \& Fischer (2005). We further constrained the surface gravity using Yonsei-Yale $\left(\mathrm{Y}^{2}\right)$ stellar structure models (Demarque et al. 2004) and revised Hipparcos parallaxes (van Leeuwen 2007) with the iterative method of Valenti et al. (2009). The resulting stellar parameters are listed in Table 1 along with the directly measured parameters from Section 2. The SME
Table 2

HD 38529 Radial Velocities ${ }^{\mathrm{a}}$

\begin{tabular}{|c|c|c|c|}
\hline $\begin{array}{l}\text { Date } \\
(\mathrm{JD}-2440000)\end{array}$ & $\begin{array}{c}\mathrm{RV} \\
\left(\mathrm{m} \mathrm{s}^{-1}\right)\end{array}$ & $\begin{array}{c}\sigma \\
\left(\mathrm{m} \mathrm{s}^{-1}\right)\end{array}$ & Data Set $^{\mathrm{b}}$ \\
\hline 13341.779899 & -105.27 & 7.77 & 1 \\
\hline 13341.898484 & -118.43 & 7.25 & 1 \\
\hline 13355.845730 & -102.05 & 7.34 & 1 \\
\hline 13357.859630 & -105.27 & 7.48 & 1 \\
\hline 13358.724097 & -87.82 & 7.11 & 1 \\
\hline 13359.729188 & -82.07 & 8.70 & 1 \\
\hline 13360.849520 & -65.85 & 7.80 & 1 \\
\hline 13365.817387 & 1.45 & 7.73 & 1 \\
\hline 13367.812640 & -20.41 & 9.48 & 1 \\
\hline 15095.967984 & 33.82 & 6.08 & 2 \\
\hline 15115.906428 & -45.13 & 6.09 & 2 \\
\hline 15141.862009 & 34.65 & 6.05 & 2 \\
\hline 15142.942977 & 2.05 & 5.64 & 2 \\
\hline 15175.755713 & -66.65 & 5.90 & 2 \\
\hline 15175.758488 & -68.20 & 5.60 & 2 \\
\hline 15176.860583 & -44.69 & 5.07 & 2 \\
\hline 15182.725710 & 38.24 & 4.68 & 2 \\
\hline 15185.724484 & -15.63 & 5.29 & 2 \\
\hline 10418.959317 & 75.60 & 1.32 & 3 \\
\hline 10545.771238 & 12.67 & 1.35 & 3 \\
\hline 10787.014317 & -69.45 & 1.40 & 3 \\
\hline 10807.061991 & -70.45 & 1.30 & 3 \\
\hline 10837.758229 & -111.32 & 1.50 & 3 \\
\hline 10838.784387 & -113.34 & 1.43 & 3 \\
\hline 10861.729653 & -30.86 & 1.42 & 3 \\
\hline 10862.725174 & -41.86 & 1.46 & 3 \\
\hline 11073.058843 & -64.92 & 1.32 & 3 \\
\hline 13750.837303 & 32.41 & 1.18 & 4 \\
\hline 13750.837905 & 32.48 & 1.32 & 4 \\
\hline 13750.839132 & 27.87 & 1.02 & 4 \\
\hline 13751.878079 & 49.46 & 0.99 & 4 \\
\hline 13752.884016 & 47.48 & 0.92 & 4 \\
\hline 13753.895081 & 33.16 & 0.98 & 4 \\
\hline 13775.747373 & -5.19 & 0.91 & 4 \\
\hline 13776.880150 & 10.45 & 0.87 & 4 \\
\hline 14336.130035 & 261.65 & 1.11 & 4 \\
\hline 11101.015625 & -101.58 & 6.57 & 5 \\
\hline 11101.035156 & -111.09 & 6.40 & 5 \\
\hline 11102.014648 & -88.30 & 6.17 & 5 \\
\hline 11102.032227 & -87.47 & 6.68 & 5 \\
\hline 11131.912109 & -91.36 & 5.99 & 5 \\
\hline 11131.930664 & -79.77 & 7.06 & 5 \\
\hline 11132.928711 & -67.59 & 6.02 & 5 \\
\hline 11154.808594 & -140.06 & 6.89 & 5 \\
\hline 11173.906250 & -104.89 & 10.69 & 5 \\
\hline 12267.809570 & 143.48 & 3.88 & 6 \\
\hline 12267.818359 & 150.79 & 3.86 & 6 \\
\hline 12298.721680 & 103.71 & 4.99 & 6 \\
\hline 12298.751953 & 113.82 & 5.33 & 6 \\
\hline 12298.785156 & 105.67 & 5.06 & 6 \\
\hline 12298.815430 & 93.78 & 6.53 & 6 \\
\hline 12299.695312 & 110.60 & 4.16 & 6 \\
\hline 12335.657227 & 206.29 & 4.74 & 6 \\
\hline 12534.970703 & 31.96 & 4.15 & 6 \\
\hline
\end{tabular}

Notes.

a The complete data set contains 436 measurements.

b Data sets 1 and 2, 3 and 4, 5 and 6 are from telescopes HET, Keck, and Lick, respectively.

(This table is available in its entirety in a machine-readable form in the online journal. A portion is shown here for guidance regarding its form and content.) 
Table 3

Keplerian Orbital Model

\begin{tabular}{|c|c|}
\hline Parameter & Value \\
\hline \multicolumn{2}{|l|}{ HD $38529 \mathrm{~b}$} \\
\hline$P$ (days) & $14.30978 \pm 0.00033$ \\
\hline$T_{c}{ }^{\mathrm{a}}(\mathrm{JD}-2,440,000)$ & $15815.633 \pm 0.063$ \\
\hline$T_{p}^{\mathrm{b}}(\mathrm{JD}-2,440,000)$ & $12281.19 \pm 0.15$ \\
\hline$e$ & $0.259 \pm 0.016$ \\
\hline$K\left(\mathrm{~m} \mathrm{~s}^{-1}\right)$ & $56.81 \pm 1.01$ \\
\hline$\omega(\operatorname{deg})$ & $93.3 \pm 4.1$ \\
\hline$M_{p} \sin i\left(M_{J}\right)$ & $0.8047 \pm 0.0139$ \\
\hline$a(\mathrm{AU})$ & $0.1278 \pm 0.0006$ \\
\hline \multicolumn{2}{|l|}{ HD $38529 \mathrm{c}$} \\
\hline$P$ (days) & $2133.54 \pm 3.31$ \\
\hline$T_{p}^{\mathrm{b}}(\mathrm{JD}-2,440,000)$ & $12264.49 \pm 6.43$ \\
\hline$e$ & $0.3472 \pm 0.0057$ \\
\hline$K\left(\mathrm{~m} \mathrm{~s}^{-1}\right)$ & $170.54 \pm 1.12$ \\
\hline$\omega(\operatorname{deg})$ & $20.08 \pm 1.14$ \\
\hline$M_{p} \sin i\left(M_{J}\right)$ & $12.51 \pm 0.08$ \\
\hline$a(\mathrm{AU})$ & $3.594 \pm 0.018$ \\
\hline \multicolumn{2}{|l|}{ System Properties } \\
\hline$\gamma\left(\mathrm{m} \mathrm{s}^{-1}\right)$ & $96.34 \pm 3.59$ \\
\hline \multicolumn{2}{|l|}{ Measurements and Model } \\
\hline$N_{\text {obs }}$ & 436 \\
\hline $\operatorname{rms}\left(\mathrm{m} \mathrm{s}^{-1}\right)$ & 11.76 \\
\hline$\chi_{\text {red }}^{2}$ & 11.67 \\
\hline
\end{tabular}

Notes.

a Time of mid-transit.

b Time of periastron passage.

derived parameters are effective temperature, surface gravity, iron abundance, projected rotational velocity, mass, radius, and age. These properties are consistent with a slightly metal-rich, mid-G sub-giant. There are other literature sources that have analyzed the abundances in HD 38529, namely: Gonzalez et al. (2001), Zhao et al. (2002), Bodaghee et al. (2003), Ecuvillon et al. (2006), Gilli et al. (2006), Takeda et al. (2005), Delgado Mena et al. (2010), Brugamyer et al. (2011), Kang et al. (2011), Petigura \& Marcy (2011). These catalogs contain [Fe/H] values measured with a variety of telescopes, techniques, and stellar models, and the determinations span the range of $0.28-0.46$ dex with a mean of 0.38 dex and a median of 0.40 dex. These findings are consistent with the results of Valenti \& Fischer (2005), where the average error for $[\mathrm{Fe} / \mathrm{H}]$ is \pm 0.05 dex.

The stellar radius is an essential parameter for estimating the depth and duration of a planetary transit. Although the spectroscopic and interferometric derived temperatures are consistent with each other, the radii are not. This difference is a result of the SME luminosity, which uses bolometric corrections. These corrections can sometimes be unreliable for evolved stars such as HD 38529 and, in this case, is underestimated compared with the luminosity derived from the SED fit and parallax. Since a similar $T_{\text {eff }}$ is found from the two techniques, this results in a reduced radius estimate. As described in Section 3.5, we adopt the interferometric radius for the purposes of estimating the predicted transit properties.

\subsection{Keplerian Orbital Solution}

We fit a two-planet Keplerian orbital solution to the RV data using the partially linearized, least-squares fitting procedure described in Wright \& Howard (2009) and estimated parameter uncertainties using the BOOTTRAN bootstrapping routines described in Wang et al. (2012). Table 3 lists the resulting
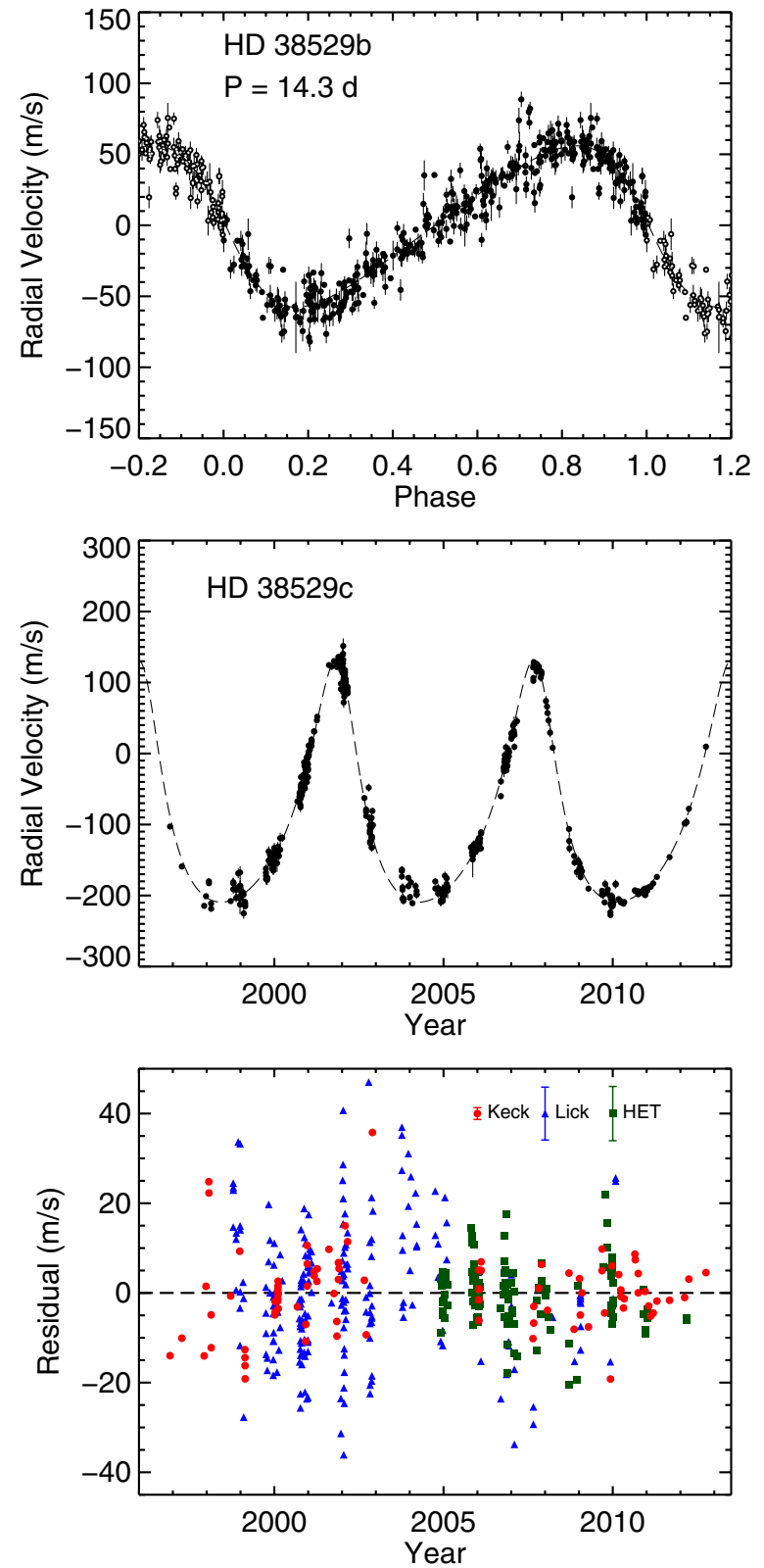

Figure 2. Top and middle panels: radial velocity signal (black dots) induced by HD 38529 b and c, respectively, and the best-fit orbital solution (dashed line). Error bars shown are internal errors for each observation. The radial velocity signal for each planet was extracted by subtracting off the best-fit orbital velocities of the other planet from the total observed RVs. Bottom panel: residual velocities with respect to the best two-planet orbital solution. The red dots are for Keck data (data sets 3 and 4), the blue triangles are for Lick data (data sets 5 and 6), and the green squares are for the HET data (data sets 1 and 2 ). The typical size of internal error bars for each telescope ( \pm median internal errors) are plotted on the upper right of this panel.

(A color version of this figure is available in the online journal.)

parameters for the two-planet orbital solution. The data and orbital solutions are shown separately for the $\mathrm{b}$ and $\mathrm{c}$ planets in Figure 2, along with the residual velocities with respect to the best two-planet orbital solution. The improved mass estimate for planet c combined with the Fine Guidance Sensor (FGS) astrometry of Benedict et al. (2010) yields a true mass estimate of $16.76 \pm 0.11 M_{J}$. As noted by Benedict et al. (2010), this lies within the brown dwarf mass regime.

The fit required five additional free parameters due to the offsets between the six independent data sets. The offsets with 


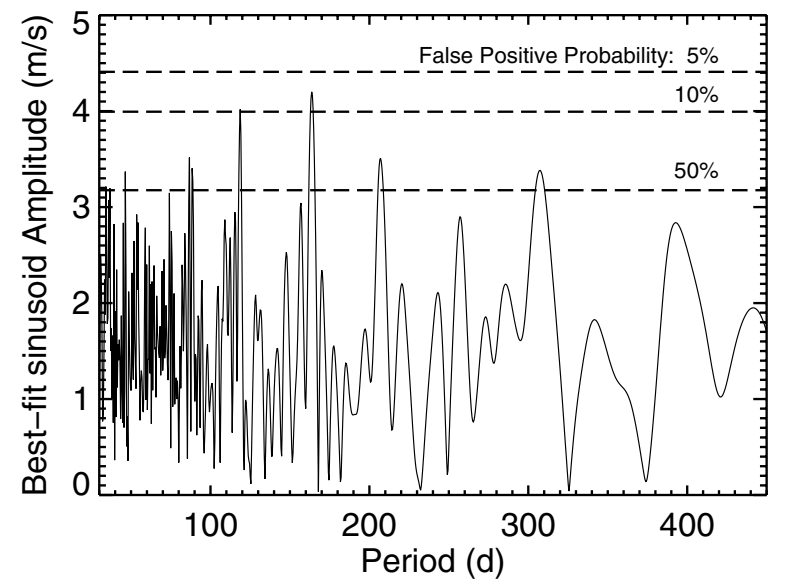

Figure 3. Amplitude of best-fit sinusoids to the residuals of the two-planet Keplerian solution (solid line). Any peak in this period window that has amplitude larger than the top dashed line is considered to be significant for having $<5 \%$ false positive probability. The two lower dashed lines $(<10 \%$ and $<50 \%$ ) have similar meanings. No period within this window has less than $5 \%$ false positive probability, and the two peaks with $<10 \%$ false positive probability are at 119 days and 164 days. We see no significant peak around 194 days as reported by Benedict et al. (2010). See Section 3.4 for more details.

respect to the observations acquired with HET data set 2 (the new HET data presented in this paper, see Section 3.1) are $-44.9,-63.92,-73.01,-117.2$, and $-123.1 \mathrm{~m} \mathrm{~s}^{-1}$ for data sets $1,3,4,5$, and 6 , respectively. As shown in Table 3 , the $\chi_{\text {red }}^{2}$ and rms scatter of the residuals are relatively large. The major contributor to the calculated $\chi_{\text {red }}^{2}$ values are the Keck data whose uncertainties are significantly smaller than those associated with the Lick and HET data. Specifically, the Keck rms scatter of $8.6 \mathrm{~m} \mathrm{~s}^{-1}$ exceeds its internal error, which has a median value of $1.3 \mathrm{~m} \mathrm{~s}^{-1}$. This indicates that there is a stellar noise component to the overall noise level which is not accounted for in the fit. The causes of the stellar noise, including pulsations and star spot activity, are discussed in detail by Benedict et al. (2010). Another hypothesis is that of an additional planet with an amplitude of $K \gtrsim 5 \mathrm{~m} \mathrm{~s}^{-1}$. We explain in the following section that our data do not support detection of such an additional planet.

\subsection{A Third Planet?}

Benedict et al. (2010) utilize their results to speculate on evidence for a third planet in the system. Thus, we also consider this possibility from our analysis since our RV data comprise a substantially larger data set. As reported by Benedict et al. (2010), a coplanar orbital solution is only stable if the third planet has a period within the window of [33, 445] days and an eccentricity of $e<0.3$, or a period larger than their RV data baseline ( $>10$ years). For this reason, we focused our search for the third planet within the period window of [33, 445] days and constrained the eccentricity to be $<0.3$.

We first searched for strong periodic signals in the residuals of the two-planet Keplerian solution by fitting sinusoids to the residuals at different periods within [33, 445] days (with 0.4 day step in period). The results are plotted as the solid line in Figure 3 . We then estimated the false positive probability to see if any of the strong peaks are significant. We define the false positive probability for a peak with a certain amplitude $K^{\prime}$ as the probability that a signal with amplitude $\geqslant K^{\prime}$ is generated by the residuals by chance. We generated 1000 sets of simulated residuals by scrambling the true residuals (and their associated errors, with replacements), and then searched for the peak with the largest amplitude within the $P=[33,445]$ day window for each of the 1000 scrambled data sets. These 1000 amplitudes provide approximately the distribution of amplitudes arising purely from random noise in the residuals. Any peak in Figure 3 that has an amplitude smaller than 950 (95\%) of these 1000 amplitudes is thus considered having false positive probability of $>5 \%$. This is marked by the top dashed line in Figure 3 and similarly for the $10 \%$ and $50 \%$ lines.

As shown in Figure 3, no peak has a false positive probability of less than $5 \%$, and there are just two with less than $10 \%$ at 119 days and 164 days. The highest peak at 164 days has a false positive probability of $6.8 \%$. We see no significant peak around 194 days as reported by Benedict et al. (2010). We then performed a three-planet Keplerian fit with our RV data within the $P=[33,445]$ day window and with the constraint that the eccentricity must be smaller than 0.3 . We found that indeed the best-fit is near 164 days, with $e=0.3$ (also true if we force the third planet to be on a circular orbit; best-fit $e=0.99$ if no constraint on $e$ is required). The $\chi_{v}^{2}$ of this fit is 9.58 , and an f-test suggests that the three-planet model provides a better fit though having 5 more parameters. However, the rms for this fit is $11.92 \mathrm{~m} \mathrm{~s}^{-1}$, i.e., adding a third planet does not reduce the rms of the fit. Combining with the fact that this signal at $P=164$ days does not have lower than 5\% false positive probability, we cannot conclude that our data have detected a third planet in the HD 38529 system.

We note here that including or excluding this third planet does not affect our transit exclusion analysis in the following sections, because the changes in the orbital parameters for both HD 38529 b or c, after adding the third planet, are smaller than their error bars listed in Table 3.

\subsection{Transit Ephemeris Refinement}

From the stellar and planetary properties listed in Tables 1 and 3, we compute a refined transit ephemeris for HD 38529b. We use the directly measured radius of the host star determined from our CHARA observations. We approximate the radius of the planet as $R_{p}=1.0 R_{J}$, based upon the mass-radius relationship described by Kane \& Gelino (2012). These properties lead to a predicted transit duration of 0.33 days and a predicted transit depth of $0.17 \%$ or 0.0018 mag. Kane \& von Braun (2008) show how the probability of a planetary transit is a strong function of both the eccentricity and the argument of periastron. The periastron argument of HD 38529 is particularly well-aligned for transit probability enhancement since it is quite close to the optimal angle of $90^{\circ}$. Combined with the eccentricity, this results in a transit probability of $12.8 \%$. However, if the planet were in a circular orbit, the duration would be increased to 0.43 days but the probability would be reduced to $9.5 \%$.

The calculation of the transit mid-point shown in Table 3 was performed with a Monte-Carlo bootstrap, which propagates the uncertainty in this orbital parameter to the time of the transit. The resulting uncertainty in the transit mid-point is 0.063 days $=$ 91 minutes. As such, the transit window is dominated by the predicted duration rather than the mid-point uncertainty, making it a suitable candidate for photometric follow-up (Kane et al. 2009). Thus, we compute a revised transit ephemeris from the orbital fit, which we utilize in the following sections.

\section{PHOTOMETRIC OBSERVATIONS}

We acquired 1106 photometric observations of HD 38529 on 992 nights between 2000 November 28 and 2012 March 
Table 4

Summary of Photometric Observations for HD 38529

\begin{tabular}{|c|c|c|c|c|c|c|c|c|}
\hline $\begin{array}{l}\text { Observing } \\
\text { Season } \\
\text { (1) }\end{array}$ & $\begin{array}{c}N_{\text {obs }} \\
\text { (2) }\end{array}$ & $\begin{array}{c}\text { Julian Date Range } \\
\text { (HJD - } 2,400,000) \\
\text { (3) }\end{array}$ & $\begin{array}{l}\text { Sigma } \\
(\mathrm{mag}) \\
(4)\end{array}$ & $\begin{array}{c}P_{\text {rot }} \\
\text { (days) } \\
(5)\end{array}$ & $\begin{array}{l}\text { Full Amplitude } \\
\text { (mag) } \\
\text { (6) }\end{array}$ & $\begin{array}{c}\langle P-C 1\rangle \\
(\mathrm{mag}) \\
(7)\end{array}$ & $\begin{array}{c}\langle P-C 2\rangle \\
(\mathrm{mag}) \\
(8)\end{array}$ & $\begin{array}{c}\langle C 2-C 1\rangle \\
(\mathrm{mag}) \\
(9)\end{array}$ \\
\hline $2000-01$ & 47 & $51877-51997$ & 0.00181 & $\ldots$ & $\cdots$ & $-1.8254 \pm 0.0002$ & $-1.8033 \pm 0.0002$ & $-0.0221 \pm 0.0001$ \\
\hline $2001-02$ & 88 & $52169-52368$ & 0.00190 & $38.5 \pm 0.5$ & $0.0024 \pm 0.0005$ & $-1.8288 \pm 0.0002$ & $-1.8061 \pm 0.0002$ & $-0.0226 \pm 0.0001$ \\
\hline $2002-03$ & 76 & $52532-52732$ & 0.00219 & $36.3 \pm 0.2^{\mathrm{a}}$ & $0.0042 \pm 0.0007$ & $-1.8303 \pm 0.0002$ & $-1.8076 \pm 0.0002$ & $-0.0226 \pm 0.0001$ \\
\hline 2003-04 & 95 & 52894-53094 & 0.00166 & $36.2 \pm 0.3$ & $0.0029 \pm 0.0004$ & $-1.8288 \pm 0.0002$ & $-1.8056 \pm 0.0001$ & $-0.0232 \pm 0.0001$ \\
\hline 2004-05 & 103 & $53258-53462$ & 0.00138 & $35.7 \pm 0.3$ & $0.0022 \pm 0.0003$ & $-1.8259 \pm 0.0001$ & $-1.8033 \pm 0.0001$ & $-0.0225 \pm 0.0001$ \\
\hline 2005-06 & 101 & $53627-53827$ & 0.00175 & $\ldots$ & $\ldots$ & $-1.8240 \pm 0.0001$ & $-1.8017 \pm 0.0001$ & $-0.0223 \pm 0.0001$ \\
\hline 2006-07 & 118 & 53996-54194 & 0.00177 & $38.0 \pm 0.2^{\mathrm{a}}$ & $0.0026 \pm 0.0004$ & $-1.8246 \pm 0.0001$ & $-1.8015 \pm 0.0001$ & $-0.0231 \pm 0.0001$ \\
\hline 2007-08 & 110 & $54370-54556$ & 0.00187 & $37.3 \pm 0.2^{\mathrm{a}}$ & $0.0021 \pm 0.0005$ & $-1.8265 \pm 0.0002$ & $-1.8037 \pm 0.0001$ & $-0.0227 \pm 0.0001$ \\
\hline 2008-09 & 104 & 54734-54919 & 0.00198 & $\ldots$ & $\ldots$ & $-1.8273 \pm 0.0002$ & $-1.8047 \pm 0.0002$ & $-0.0226 \pm 0.0001$ \\
\hline 2009-10 & 86 & $55092-55284$ & 0.00189 & $36.5 \pm 0.3$ & $0.0034 \pm 0.0006$ & $-1.8275 \pm 0.0002$ & $-1.8042 \pm 0.0001$ & $-0.0232 \pm 0.0001$ \\
\hline $2010-11$ & 95 & $55459-55650$ & 0.00184 & $\ldots$ & $\ldots$ & $-1.8253 \pm 0.0001$ & $-1.8022 \pm 0.0002$ & $-0.0230 \pm 0.0001$ \\
\hline $2011-12$ & 83 & $55830-56018$ & 0.00157 & $37.2 \pm 0.4$ & $0.0024 \pm 0.0004$ & $-1.8252 \pm 0.0002$ & $-1.8019 \pm 0.0001$ & $-0.0232 \pm 0.0001$ \\
\hline
\end{tabular}

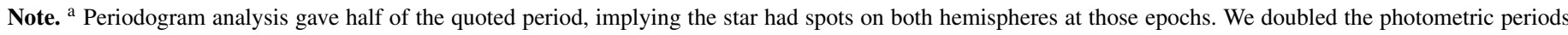
and their errors in these cases to get $P_{\text {rot }}$.

31, all with the T11 $0.80 \mathrm{~m}$ APT at Fairborn Observatory in Arizona. The T11 APT, one of several such telescopes operated at Fairborn by Tennessee State University, is equipped with a two-channel precision photometer that uses a dichroic filter and two EMI 9124QB bi-alkali photomultiplier tubes to separate and simultaneously measure the Strömgren $b$ and $y$ pass bands. We programmed the APT to make differential brightness measurements of our program star HD $38529(\mathrm{P}, V=5.95$, $B-V=0.77$, G4 IV) with respect to the two comparison stars HD $38145(\mathrm{C} 1, V=7.89, B-V=0.33, \mathrm{~F} 0 \mathrm{~V})$ and HD 40259 $(\mathrm{C} 2, V=7.86, B-V=0.38$, F0 V). From the raw counts in both pass bands, we compute the differential magnitudes $P-C 1, P-C 2$, and $C 2-C 1$, correct them for atmospheric extinction, and transform them to the Strömgren system. To improve our photometric precision, we combine the differential $b$ and $y$ observations into a single $(b+y) / 2$ passband, which we indicate with the subscript by. Furthermore, we compute the differential magnitudes of HD 38529 against the mean brightness of the two comparison stars. The resulting precision of the individual $P-(C 1+C 2) / 2_{b y}$ differential magnitudes ranges between $\sim 0.0010$ mag and $\sim 0.0015$ mag on good nights, as determined from the nightly scatter in the $C 2-C 1$ differential magnitudes of the constant comparison stars. Further details of our automatic telescopes, precision photometers, and observing and data reduction procedures can be found in Henry (1999) and Eaton et al. (2003) and references therein.

The resulting 1106 differential magnitudes, spanning 12 consecutive observing seasons, are summarized in Table 4 and plotted in the top panel of Figure 4, after normalization to bring the seasonal means to a common level indicated by the horizontal line in the top panel. The normalization removes season-to-season variability in HD 38529 perhaps caused by a starspot cycle in this mildly active star (see below). The scatter in the normalized data from their grand mean is $\sigma=0.0019 \mathrm{mag}$ (standard deviation). This is slightly larger than our typical measurement precision given above, suggesting low-amplitude, night-to-night variability in HD 38529.

The normalized observations from all 12 observing seasons are replotted in the middle panel of Figure 4, phased with the 14.3 day planetary orbital period of HD $38529 \mathrm{~b}$ and the time of mid transit $\left(T_{c}\right)$ from Table 3. A least-squares sinusoidal fit to the phased data gives a formal semi-amplitude of just $0.00014 \pm 0.00007 \mathrm{mag}$, which limits any periodic brightness
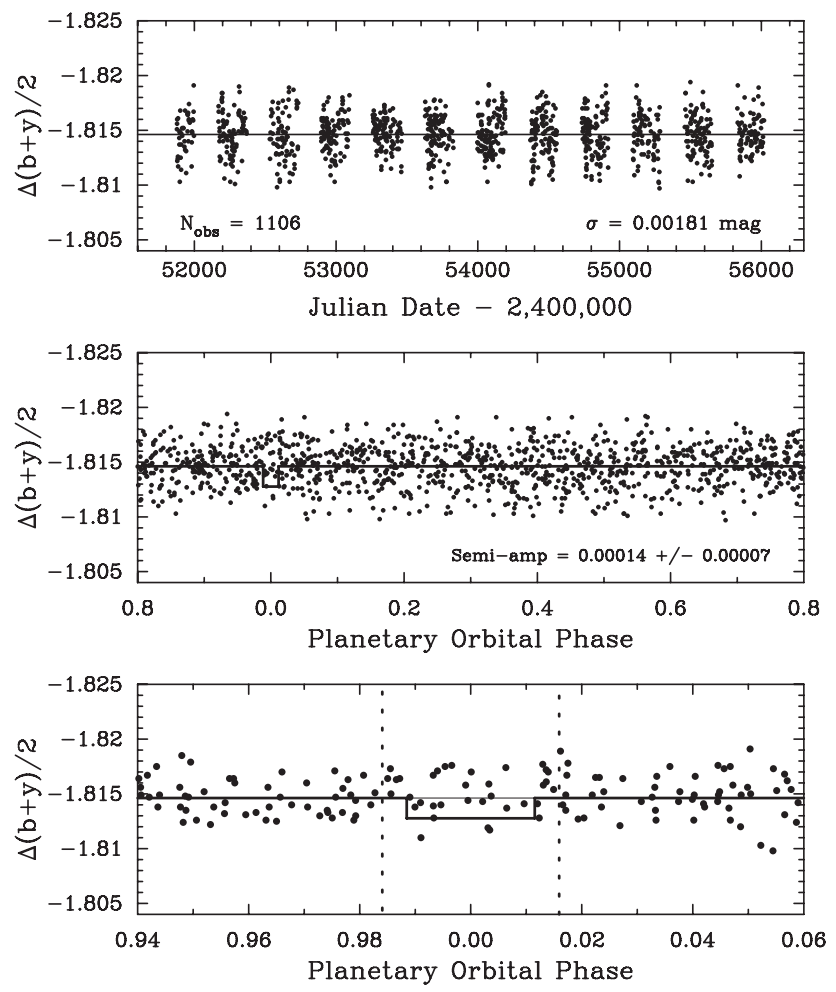

Figure 4. Top: the 1106 individual $P-(C 1+C 2) / 2_{b y}$ differential magnitudes of HD 38529, acquired with the T11 $0.8 \mathrm{~m}$ APT during 12 observing seasons between 2000 and 2012. The observations in each observing season have been normalized to give each observing season the same yearly mean. Middle: the 1106 observations phased with the orbital period and time of transit of companion b. The semi-amplitude of a least-squares sine fit to this orbital phase curve is $0.00014 \pm 0.00007 \mathrm{mag}$, consistent with the absence of periodic light variability in HD 38529 on the radial velocity period. This provides strong confirmation of the existence of planet $b$. Bottom: the observations near phase 0.0 plotted on an expanded scale. The solid curve shows the estimated depth $(\sim 0.0018 \mathrm{mag})$ and duration $( \pm 0.012$ phase units $)$ of a central transit of companion $\mathrm{b}$. The $\pm 1 \sigma$ extent of the transit window is indicated by the vertical dotted lines. Transits are ruled out to a depth of $\sim 0.0004$ mag.

variability of the star on the orbital period to a very small fraction of $1 \mathrm{mmag}$. This rules out the possibility that the 14.3 day radial velocity variations are simply jitter induced by stellar activity, as has been documented in slightly more active stars, for instance, by Queloz et al. (2001), Paulson et al. (2004), and Boisse et al. 
(2012). Instead, the lack of photometric variability confirms that the radial velocity variations in HD 38529 result from true planetary reflex motion.

The photometric observations within \pm 0.06 phase units of mid-transit are plotted with an expanded scale in the lower panel of Figure 4 . The solid curve shows predicted transit phase $(0.0)$, depth $(\sim 0.0018 \mathrm{mag})$, and duration $( \pm 0.012$ phase units $)$ of a central transit, all computed from the stellar radius in Table 1 and the orbital elements of HD 38529b in Table 3. The vertical dotted lines give the $\pm 1 \sigma$ uncertainty in the timing of the transit window, based on the uncertainties in the stellar radius and the improved orbital elements from Tables 1 and 3, respectively. Our data set contains 1084 photometric observations that lie outside the predicted transit time (solid curve); these have a mean of $-1.81462 \pm 0.00005$ mag. There are 22 observations that fall in transit; these have a mean of $-1.81474 \pm 0.00041 \mathrm{mag}$. The difference is our "observed transit depth," $-0.00012 \pm 0.00042 \mathrm{mag}$ in the sense that the mean of the transit points is slightly brighter than the mean of the out-of-transit observations but are, nevertheless, consistent with zero to four decimal places. As the lower panel shows, we have sufficient data around predicted transit times to rule out transits to a depth of $\sim 0.0004 \mathrm{mag}$.

It has been suggested by Anglada-Escudé et al. (2010) that planetary systems in 2:1 orbital resonance can be mistaken for a single planet in an eccentric orbit. If planet $b$ has an orbital period that is half of the derived $\sim 14.3$ day period, then it would have an even higher transit probability and thus a higher chance of being detected in the photometric data. We performed a search through the 12 year photometric data set for periodic transits around 7.155 days and around 14.3 days. No transits were detected at or near either period. Although a dynamical model of a 2:1 resonance would lead to aperiodic transit times, the complete phase coverage of our photometric observations over a range of periods around 14.3 days leads us to conclude that such transits are ruled out.

As noted above, the scatter in the normalized data set is slightly larger than the expected measurement precision, suggesting the presence of small starspots on HD 38529. Starspots on the photospheres of solar-type stars allow the possibility of direct determination of stellar rotation periods from rotational modulation in the visibility of the spots and the consequent variability in the star's brightness (see, e.g., Simpson et al. 2010). Spots can also produce periodic radial velocity variations that can mimic the presence of a planetary companion. Therefore, we performed periodogram analyses for each of the 12 seasons of normalized photometry plotted in the top panel of Figure 4 and, indeed, found very low-amplitude periodic brightness variations in 8 of the 12 observing seasons. Similar analyses of the twelve $C 2-C 1$ seasonal data sets evinced no significant periodicity in the comparison stars. Figure 5 shows a sample frequency spectrum and phase curve for Season 5. Complete results are given in Columns 5 and 6 of Table 4 . The seasonal photometric periods scatter about their mean with a standard deviation of $\sim 1$ day. The weighted mean of the 8 photometric periods is $37.0 \pm 0.4$ days, which we take to be our best determination of the star's rotation period. This period is based on far more observations than the preliminary periods of 35.7 and 31.6 days given by Fischer et al. (2003) and Benedict et al. (2010), respectively. The seasonal peak-to-peak amplitudes in Column 6 range between 0.002 to $0.004 \mathrm{mag}$, indicating spot filling factors of only a few tenths of one percent. Both the low level of spottedness and the fact that the stellar rotation period is
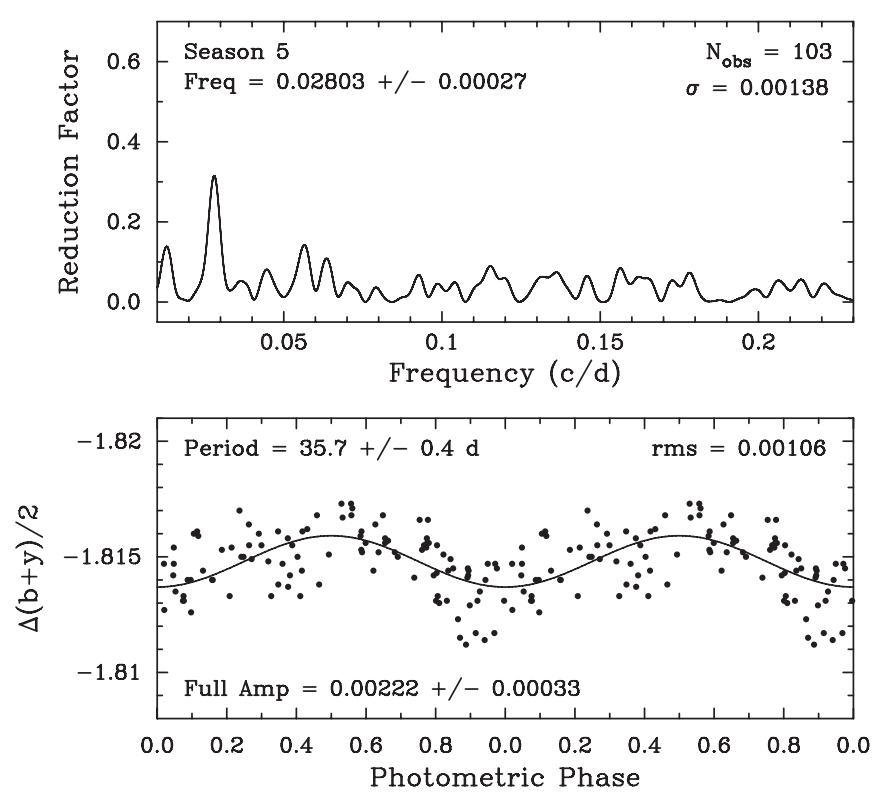

Figure 5. Top: a frequency spectrum of the 2004-2005 (Season 5) photometric observations of HD 38529. The best frequency occurs at $0.02803 \pm 0.00027$ cycles per day. Bottom: the 103 Season 5 observations phased with the corresponding best period of 35.7 days. The phase curve shows coherent variability with a peak-to-peak amplitude of 0.002 mag, which we take to be rotational modulation of photospheric spots. Eight of the twelve observing seasons exhibit similar modulation (see Table 4).

distinctly different from the 14 day radial velocity period and its harmonics demonstrates that stellar activity (spots and plages) is not the source of the 14 day radial velocity period.

If the inclination of HD 38529's rotation axis is near $90^{\circ}$, so that $V \sin i$ approximately equals the equatorial rotation velocity, $V_{\mathrm{eq}}$, then the stellar radius and projected rotational velocity from Table 1 result in a rotation period of $\sim 37.5$ days, essentially identical to our observed value of $P_{\text {rot }}=37.0$ days. This implies a very high inclination for the stellar rotation axis and increases the probability of transits of HD 38529b, assuming the stellar equatorial and planetary orbital planes are aligned. Moutou et al. (2011) have shown, for instance, that the orbital planes of the hot Jupiters HAT-P-8b, HAT-P-9b, HAT-P-16b, \& HAT-P-23b are all closely aligned with the stellar rotation axis. In the same paper, Moutou et al. (2011) examined 37 exoplanetary systems that have accurately measured spinorbit angles and found that spin-orbit misalignment occurs primarily for stars with $T_{\text {eff }}>6300 \mathrm{~K}$ (spectral class F6 and hotter). Thus, the orbital geometry, the high stellar inclination, and the likelihood of spin-orbit alignment are all favorable for transits of HD 38529b, making our non-detection particularly disappointing.

Finally, we examine long-term variability in HD 38529's Ca II $\mathrm{H}$ and $\mathrm{K}$ indices and APT photometry to look for evidence of magnetic cycles that might induce apparent radial velocity variations and so mimic the presence of a long-period planet. Both $\mathrm{H}$ and $\mathrm{K}$ emission and brightness variability are good proxies for stellar magnetic activity (see, e.g., Baliunas et al. 1995, 1998; Lockwood et al. 2007, and references therein). In the top panel of Figure 6, we plot seasonal means of the Mount Wilson S-index derived from our Keck I RV spectra as described in Wright et al. (2004) and Isaacson \& Fischer (2010). Despite a couple of observing seasons without Keck H and $\mathrm{K}$ measurements, clear variability is seen on a timescale of several years. In panels 2 and 3, we plot the 12 seasonal mean 

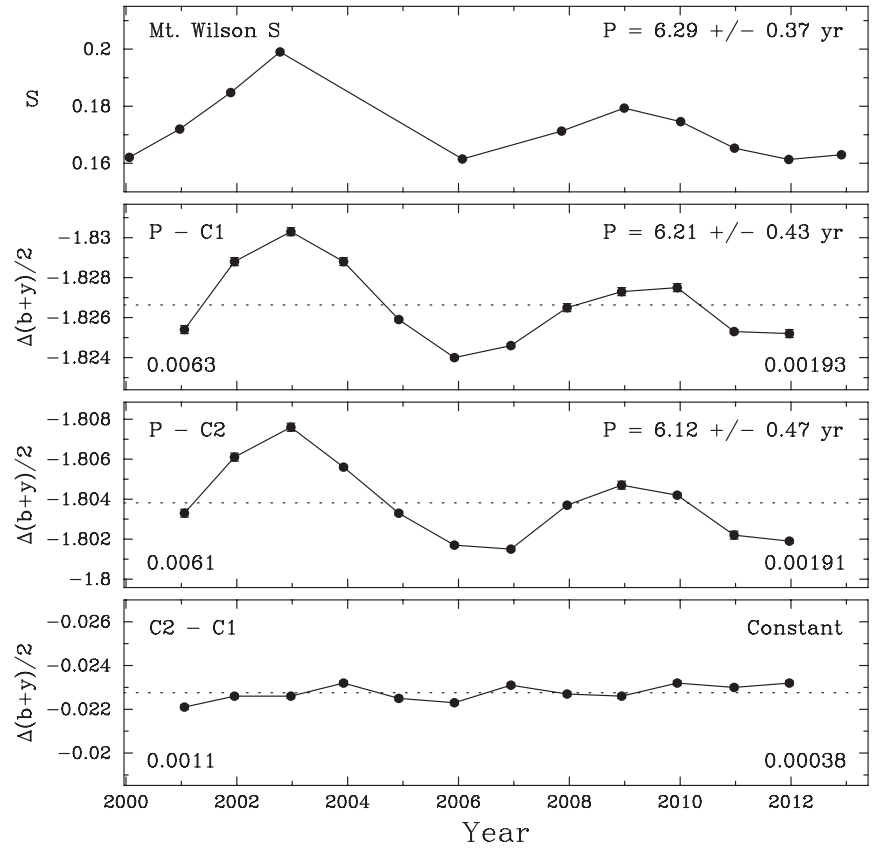

Figure 6. Long-term variations in Mt. Wilson S-index (top) and brightness (panels 2 and 3) measured with Keck I and the T11 APT. The cycle timescale is $\sim 6$ yrs in all three timeseries. Long-term differential magnitudes of the two comparison stars $\mathrm{C} 1$ and $\mathrm{C} 2$ (bottom) show excellent stability to $\pm 0.00038 \mathrm{mag}$ ), demonstrating that the variability in the $P-C 1$ and $P-C 2$ light curves is intrinsic to HD 38529. The direct correlation of S-index and brightness is typical for stars of solar age and older. The weak magnetic activity in HD 38529 cannot produce the large-amplitude radial velocity variations attributed to planet $c$.

$P-C 1$ and $P-C 2$ differential magnitudes (Table 4) without any normalization, as was applied to the $P-(C 1+C 2) / 2$ differential magnitudes in Figure 4. These two light curves show that HD 38529 varies in brightness by several mmag from year-to-year with respect to both comparison stars $\mathrm{C} 1$ and $\mathrm{C} 2$. The bottom panel of Figure 6 plots the yearly mean $C 2-C 1$ comparison star differential magnitudes. The number in the lower left corner of each panel gives the range of the seasonal means in magnitudes; the number in the lower right corner gives the standard deviation of the seasonal means with respect to their grand mean, shown by the horizontal dotted line in each panel. The standard deviation of the $C 2-C 1$ observations is only $0.00038 \mathrm{mag}$, indicating that both comparison stars have excellent long-term photometric stability. Therefore, the shortand long-term variability evident in the $P-C 1$ and $P-C 2$ light curves must be intrinsic to HD 38529.

We see cyclic variation in both the $\mathrm{H}$ and $\mathrm{K}$ and APT observations plotted in Figure 6. Simple least-squares, sine-fit periodogram analyses of the $\mathrm{H}$ and $\mathrm{K}, P-C 1$, and $P-C 2$ means give periods of $6.29 \pm 0.37,6.21 \pm 0.43$, and $6.12 \pm$ 0.47 years, respectively, all identical within their uncertainties. Furthermore, the brightness of the star and the strength of the $\mathrm{H}$ and $\mathrm{K}$ emission vary directly in phase with each other. The cycle timescale and amplitudes of the $\mathrm{H}$ and $\mathrm{K}$ and photometric observations are typical of similar long-term cycles seen in a large sample of solar-type stars being measured with the APTs (see Henry 1999; Lockwood et al. 2007; Hall et al. 2009) and are analogous to solar irradiance variations measured for over three decades with space-based radiometers (see, e.g., Willson $\&$ Hudson 1981; Frohlich 2012). The direct correlation of longterm brightness and magnetic activity in HD 38529 is in the same sense as the correlation between solar irradiance and
S-index. Despite the similarities in age and spectral class of the Sun (4.6 Gyr, G2) and HD 38529 (4.4 Gyr, G5), the amplitudes of the S-index and brightness variability are larger in HD 38529 by factors of approximately 2 and 5 , respectively (see, e.g., Lockwood et al. 2007, their Figure 2). This is due to the fact that HD 38529 is a more massive, evolved subgiant (Table 1) with a deeper convection zone.

The radial velocity period of HD 38529 c is 2134.9 days (Table 3 ) or 5.85 years, very close to the spot-cycle timescale of 6.2 years. This raises the question that the radial velocity variations attributed to HD 38529c may, instead, originate from the stellar magnetic cycle. As mentioned above, starspots in active stars have been found to induce radial velocity variations on stellar rotation timescales. Over the past 15 years or so, several authors have discussed the mechanisms by which longterm (decadal) magnetic cycles might be the source of longterm radial velocity variations (e.g., Saar \& Donahue 1997; Saar \& Fischer 2000; Santos et al. 2010). Recently, Lovis et al. (2011) analyzed a sample of 304 FGK stars from the HARPS high-precision planet-search sample, looking for possible correlations of stellar activity cycles with radial velocity and spectral line-shape parameters. They confirmed that stellar magnetic cycles can induce long-period, low-amplitude radial velocity variations with amplitudes up to $25 \mathrm{~m} \mathrm{~s}^{-1}$ in the more active stars. For HD 38529c, however, its radial velocity semiamplitude of $171 \mathrm{~m} \mathrm{~s}^{-1}$ makes it immune to this type of false positive.

\section{CONCLUSIONS}

With the discovery of extrasolar planets, characterization has become an important pursuit and is intrinsically linked to the understanding of the host star properties. Thus, even relatively bright stars, such as HD 38529, are worthy of further scrutiny in order to gain insight into properties of the planets hosted by the star. Here we have performed just such a task by presenting the most accurate properties of the star HD 38529 so far produced. Direct measurements of stellar radii via interferometry provide an essential test of stellar models and validation of radii derived from spectroscopy. In this case, we measure a radius of $2.578 R_{\odot}$ for HD 38529, which is consistent with a slightly metal-rich midG sub-giant. The new RV data for the star are used to calculate a new Keplerian orbital solution for the system that enables us to place significant limits on a previously postulated third planet. Combining our mass estimate for the c component with the FGS astrometry of Benedict et al. (2010) allows us to confirm that this object does lie within the brown dwarf mass regime. The refined transit ephemeris from the Keplerian orbital solution is combined with 12 years of precision photometry to demonstrate the variability of the host star and the dispositive null detection of transits for HD 38529b. The importance of this study is clear since the combination and inter-dependence of all these effects leads to a greatly improved understanding of the system as a whole. The TERMS project is systematically proceeding to provide such characterization of the planetary systems known to orbit the brightest stars in the sky.

The Center for Exoplanets and Habitable Worlds is supported by the Pennsylvania State University, the Eberly College of Science, and the Pennsylvania Space Grant Consortium. S.R.K. and N.R.H. acknowledge financial support from the National Science Foundation through grant AST-1109662. G.W.H. acknowledges support from NASA, NSF, Tennessee State University, and the State of Tennessee through its Centers of Excellence 
program. The HET is a joint project of the University of Texas at Austin, the Pennsylvania State University, Stanford University, Ludwig-Maximilians-Universität München, and Georg-AugustUniversität Göttingen. The HET is named in honor of its principal benefactors, William P. Hobby and Robert E. Eberly.

\section{REFERENCES}

Anglada-Escudé, G., López-Morales, M., \& Chambers, J. E. 2010, ApJ, 709,168

Argue, A. N. 1966, MNRAS, 133, 475

Baines, E. K., McAlister, H. A., ten Brummelaar, T. A., et al. 2008, ApJ, 680,728

Bakos, G., Noyes, R. W., Kovács, G., et al. 2004, PASP, 116, 266

Baliunas, S. L., Donahue, R. A., Soon, W. H., et al. 1995, ApJ, 438, 269

Baliunas, S. L., Donahue, R. A., Soon, W., \& Henry, G. W. 1998, in ASP Conf. Ser. 154, The 10th Cambridge Workshop on Cool Stars, Stellar Systems, and the Sun, ed. R. A. Donahue \& J. A. Bookbinder (San Francisco, CA: ASP), 153

Benedict, G. F., McArthur, B. E., Bean, J. L., et al. 2010, ApJ, 139, 1844

Bodaghee, A., Santos, N. C., Israelian, G., \& Mayor, M. 2003, A\&A, 404, 715

Boisse, I., Bonfils, X., \& Santos, N. C. 2012, A\&A, 545, A109

Borucki, W. J., Koch, D. G., Basri, G., et al. 2011a, ApJ, 728, 117

Borucki, W. J., Koch, D. G., Basri, G., et al. 2011b, ApJ, 736, 19

Boyajian, T. S., McAlister, H. A., van Belle, G., et al. 2012a, ApJ, 746, 101

Boyajian, T. S., von Braun, K., van Belle, G., et al. 2012b, ApJ, 757, 112

Brugamyer, E., Dodson-Robinson, S. E., Cochran, W. D., \& Sneden, C. 2011, ApJ, 738, 97

Charbonneau, D., Brown, T. M., Latham, D. W., \& Mayor, M. 2000, ApJL, 529, L45

Claret, A. 2000, A\&A, 363, 1081

Cousins, A. W. J. 1962, MNSSA, 21, 20

Cutri, R. M., Skrutskie, M. F., van Dyk, S., et al. 2003, The IRSA 2MASS All-Sky Point Source Catalog, NASA/IPAC Infrared Science Archive

Delgado Mena, E., Israelian, G., González Hernández, J. I., et al. 2010, ApJ, 725,2349

Demarque, P., Woo, J., Kim, Y., \& Yi, S. K. 2004, ApJS, 155, 667

Dragomir, D., Kane, S. R., Henry, G. W., et al. 2012, ApJ, 754, 37

Eaton, J. A., Henry, G. W., \& Fekel, F. C. 2003, in The Future of Small Telescopes in the New Millennium, Vol. II, The Telescopes We Use, ed. T. D. Oswalt (Dordrecht: Kluwer), 189

Ecuvillon, A., Israelian, G., Santos, N. C., Mayor, M., \& Gilli, G. 2006, A\&A, 449, 809

Fischer, D. A., Marcy, G. W., Butler, R. P., et al. 2001, ApJ, 551, 1107

Fischer, D. A., Marcy, G. W., Butler, R. P., et al. 2003, ApJ, 586, 1394

Frohlich, C. 2012, SGeo, 33, 453

Gilli, G., Israelian, G., Ecuvillon, A., Santos, N. C., \& Mayor, M. 2006, A\&A, 449,723

Golay, M. 1972, VA, 14, 13

Gonzalez, G., Brownlee, D., \& Ward, P. 2001, Icar, 152, 185

Hall, J. C., Henry, G. W., Lockwood, G. W., Skiff, B. A., \& Saar, S. H. 2009, AJ, 138,312
Hanbury Brown, R., Davis, J., Lake, R. J. W., \& Thompson, R. J. 1974, MNRAS 167,475

Hauck, B., \& Mermilliod, M. 1998, A\&AS, 129, 431

Henry, G. W. 1999, PASP, 111, 845

Henry, G. W., Marcy, G. W., Butler, R. P., \& Vogt, S. S. 2000, ApJL, 529, L41

Isaacson, H., \& Fischer, D. 2010, ApJ, 725, 875

Johnson, H. L., Mitchell, R. I., Iriarte, B., \& Wisniewski, W. Z. 1966, CoLPL, 4, 99

Kane, S. R., \& Gelino, D. M. 2012, PASP, 124, 323

Kane, S. R., Mahadevan, S., von Braun, K., Laughlin, G., \& Ciardi, D. R. 2009, PASP, 121, 1386

Kane, S. R., \& von Braun, K. 2008, ApJ, 689, 492

Kang, W., Lee, S.-G., \& Kim, K.-M. 2011, ApJ, 736, 87

Lockwood, G. W., Skiff, B. A., Henry, G. W., et al. 2007, ApJS, 171, 260

Lovis, C., et al. 2011, A\&A, submitted (arXiv:1107.5325)

McClure, R. D., \& Forrester, W. T. 1981, PDAO, 15, 439

Mermilliod, J.-C. 1986, Catalogue of Eggen's UBV Data

Moutou, C., Daz, R. F., Udry, S., et al. 2011, A\&A, 533, A113

Olsen, E. H. 1993, A\&AS, 102, 89

Paulson, D. B., Saar, S. H., Cochran, W. D., \& Henry, G. W. 2004, AJ, 127,1644

Petigura, E. A., \& Marcy, G. W. 2011, ApJ, 735, 41

Pickles, A. J. 1998, PASP, 110, 863

Pollacco, D. L., Skillen, I., Collier Cameron, A., et al. 2006, PASP, 118, 1407

Queloz, D., Henry, G. W., Sivan, J. P., et al. 2001, A\&A, 379, 279

Saar, S. H., \& Donahue, R. A. 1997, ApJ, 485, 319

Saar, S. H., \& Fischer, D. 2000, ApJL, 534, L105

Santos, N. C., da Silva, J. G., Lovis, C., \& Melo, C. 2010, A\&A, 511, A54

Simpson, E. K., Baliunas, S. L., Henry, G. W., \& Watson, C. A. 2010, MNRAS, 408, 1666

Sturmann, J., ten Brummelaar, T. A., Ridgway, S. T., et al. 2003, Proc. SPIE, 4838, 1208

Takeda, Y., Hashimoto, O., Taguchi, H., et al. 2005, PASJ, 57, 751

ten Brummelaar, T. A., McAlister, H. A., Ridgway, S. T., et al. 2005, ApJ, 628,453

Tull, R. G. 1998, Proc. SPIE, 3355, 387

Valenti, J. A., \& Fischer, D. A. 2005, ApJS, 159, 141

Valenti, J. A., Fischer, D., Marcy, G. W., et al. 2009, ApJ, 702, 989

Valenti, J. A., \& Piskunov, N. 1996, A\&AS, 118, 595

van Leeuwen, F. 2007, A\&A, 474, 653

Vogt, S. S. 1987, PASP, 99, 1214

Vogt, S. S., Allen, S. L., Bigelow, B. C., et al. 1994, Proc. SPIE, 2198, 362

von Braun, K., Boyajian, T. S., Kane, S. R., et al. 2011a, ApJL, 729, L26

von Braun, K., Boyajian, T. S., Kane, S. R., et al. 2012, ApJ, 753, 171

von Braun, K., Boyajian, T. S., ten Brummelaar, T. A., et al. 2011b, ApJ, 740,49

Wang, S. X., Wright, J. T., Cochran, W., et al. 2012, ApJ, 761, 46

Willson, R. C., \& Hudson, H. S. 1981, ApJL, 244, L185

Wittenmyer, R. A., Endl, M., Cochran, W. D., Levison, H. F., \& Henry, G. W. 2009, ApJS, 182, 97

Wright, J. T., \& Howard, A. W. 2009, ApJS, 182, 205

Wright, J. T., Marcy, G. W., Butler, R. P., \& Vogt, S. S. 2004, ApJS, 152,261

Wright, J. T., Upadhyay, S., Marcy, G. W., et al. 2009, ApJ, 693, 1084

Zhao, G., Chen, Y. Q., Qiu, H. M., \& Li, Z. W. 2002, AJ, 124, 2224 